\title{
A potential role for skeletal muscle caveolin-1 as an insulin sensitivity modulator in ageing-dependent non-obese type 2 diabetes: studies in a new mouse model
}

\author{
Y. S. Oh • L.-Y. Khil • K. A. Cho • S. J. Ryu • M. K. Ha • \\ G. J. Cheon • T. S. Lee • J.-W. Yoon • H.-S. Jun • \\ S. C. Park
}

Received: 14 February 2008 / Accepted: 21 February 2008 /Published online: 12 April 2008

(C) Springer-Verlag 2008

\begin{abstract}
Aims/hypothesis Type 2 diabetes mellitus is a common agedependent disease. We discovered that male offspring of non-diabetic C57BL/6 and DBA/2 mice, called JYD mice, develop type 2 diabetes when they grow old. JYD mice show
\end{abstract}

Y. S. Oh and L.-Y. Khil contributed equally to this study.

J.-W. Yoon passed away on 6 April 2006.

Electronic supplementary material The online version of this article (doi:10.1007/s00125-008-0993-0) contains supplementary material, which is available to authorised users.

Y. S. Oh · S. J. Ryu $\cdot$ M. K. Ha $\cdot$ S. C. Park $(\bowtie)$

Department of Biochemistry and Molecular Biology,

Aging and Apoptosis Research Center,

Seoul National University College of Medicine,

28 Yungon Dong, Chongno Ku,

Seoul 110-799, South Korea

e-mail: scpark@snu.ac.kr

L.-Y. Khil · J.-W. Yoon $\cdot$ H.-S. Jun

Department of Microbiology and Infectious Diseases,

Faculty of Medicine, University of Calgary,

Calgary, Alberta, Canada

K. A. Cho

Department of Biochemistry,

Chonnam National University Medical School,

Kwangju, South Korea

G. J. Cheon • T. S. Lee

Division of Nuclear Medicine and RI Application,

Korea Institute of Radiological and Medical Sciences,

Seoul, South Korea

Y. S. Oh $\cdot$ H.-S. Jun $(\bowtie)$

Lee Gil Ya Cancer and Diabetes Institute,

Gachon University of Medicine and Science,

7-45 Sondo-dong, Yeonsu-ku,

Incheon 406-840, South Korea

e-mail: hsjeon@gachon.ac.kr characteristics of insulin resistance, hyperglycaemia and hyperinsulinaemia in old age without obesity. We postulated that the mechanism of age-dependent type 2 diabetes in this model relates to caveolin-1 status in skeletal muscle, which appears to regulate insulin sensitivity in the mice.

Methods We compared insulin sensitivity in aged C57BL/6 and JYD mice using glucose and insulin tolerance tests and ${ }^{18} \mathrm{~F}$ fluorodeoxyglucose positron emission tomography. We also determined insulin signalling molecules and caveolin proteins using western blotting, and altered caveolin-1 levels in skeletal muscle of C57BL/6 and JYD mice using viral vector systems, to examine the effect of this on insulin sensitivity.

Results In 30-week-old C57BL/6 and JYD mice, the basal levels of IRS-1, Akt and peroxisome proliferator-activated receptor- $\gamma$ decreased, as did insulin-stimulated phosphorylation of Akt and insulin receptor $\beta$. However, caveolin- 1 was only increased about twofold in 30-week-old JYD mice as compared with 3-week-old mice, whereas an eightfold increase was seen in $\mathrm{C} 57 \mathrm{BL} / 6$ mice. Downregulation of caveolin-1 production in C57BL/6 mice caused severe impairment of glucose and insulin tolerance. Upregulation of caveolin-1 in aged diabetic JYD mice significantly improved insulin sensitivity with a concomitant increase of glucose uptake in the skeletal muscle. Conclusions/interpretation The level of skeletal muscle caveolin-1 is correlated with the progression of age-dependent type 2 diabetes in JYD mice.

Keywords Age-dependent type 2 diabetes · Caveolin · JYD . Skeletal muscle
Abbreviations
FDG fluorodeoxyglucose
GFP green fluorescent protein
GLUT-4 glucose transporter 4 
IR insulin receptor

MES 2-( $N$-morpholino)ethanesulfonic acid

PET positron emission tomography

PKB protein kinase $\mathrm{B}$

PPAR peroxisome proliferator-activated receptor

shRNA short-hairpin RNA

\section{Introduction}

Insulin resistance plays a major role in the development of type 2 diabetes [1]. Skeletal muscle is the predominant site for disposal of glucose and fatty acids, and insulin resistance of this tissue has been recognised as the beginning of type 2 diabetes.

The incidence of type 2 diabetes increases with age [2]. In Korea, 70 to $80 \%$ of age-related, type 2 diabetic patients are non-obese, which is different from Western type 2 diabetic patients, the majority of whom are obese [3]. However, the precise role of ageing in the development of type 2 diabetes remains undefined. One reason for this may be the lack of an adequate animal model exhibiting an age-dependent nonobese phenotype of type 2 diabetes. Previous animal models such as the $o b / o b$ mouse and OLETF rat develop early-onset diabetes resembling obesity-dependent human diabetes, but not late-onset, non-obese type 2 diabetes $[4,5]$. We found that male offspring of non-diabetic $\mathrm{C} 57 \mathrm{BL} / 6$ and $\mathrm{DBA} / 2$ mice, called here JYD mice, developed type 2 diabetes when they grew old. These JYD mice developed insulin resistance, hyperglycaemia and hyperinsulinaemia in old age, but without obesity. As such, JYD mice offer the potential for investigating mechanisms related to non-obese, age-related type 2 diabetes.

Caveoli are 50 to 100 -nm-sized invaginations in cells that contain 21 to $24 \mathrm{kDa}$ structural proteins called caveolins [6]. Caveolin-1 and -2 are co-expressed in many cell types to form a hetero-oligomer in the plasma membrane [7], whereas the production of caveolin-3 is muscle-specific and regulates myotubule formation [8]. Recently, it was reported that caveolin-1 plays an important role in the regulation of glucose homeostasis, especially in adipocytes $[9,10]$. We also found that caveolin-1 plays an important role in the senescent phenotype $[11,12]$ and that caveolin-1 production is upregulated in tissues of old rats as compared with tissues from young rats [13]. It was reported that caveolin-1 is also produced in skeletal muscle [14-16] and that caveolin-1 interacts with caveolin-3 in a muscle-specific manner [17]. When we examined the functional significance of caveolin-1 and -3 in skeletal muscle, we found that caveolin-1, but not caveolin-3 plays a crucial role in glucose uptake in differentiated muscle cells [18].

With discovery of the JYD mouse model of non-obese type 2 diabetes, we decided to examine the possible involvement of skeletal muscle caveolin in the development of age-related insulin resistance in non-obese type 2 diabetes.

\section{Methods}

Animals $\mathrm{C} 57 \mathrm{BL} / 6$ and $\mathrm{DBA} / 2$ mice were obtained from Charles River Laboratories (Wilmington, MA, USA) and $o b /$ $o b$ mice were purchased from The Jackson Laboratory (Bar Harbor, ME, USA). Female C57BL/6 mice were mated with male DBA/2 mice at the University of Calgary to obtain F1 mice (JYD mice).

Mice were given free access to food and water, except when noted otherwise, and were kept on a $12 \mathrm{~h}$ light, $12 \mathrm{~h}$ dark cycle. Body weights and blood glucose levels were measured in JYD mice at 10 and 30 weeks of age. Animals with blood glucose level higher than $13.8 \mathrm{mmol} / \mathrm{l}$ were considered to be diabetic. Age-matched male C57BL/6 and DBA mice were used as controls. All protocols for animal use and killing were reviewed and approved by the Animal Care Committee of University of Calgary and Seoul National University College of Medicine.

Cells L6 cells, a rat skeletal muscle cell line, were obtained from ATCC (Manassas, VA, USA) and maintained in DMEM/ F12 containing $10 \%$ (vol./vol.) fetal bovine serum, $100 \mathrm{U} / \mathrm{ml}$ penicillin and $100 \mu \mathrm{g} / \mathrm{ml}$ streptomycin.

Glucose and insulin tolerance tests For glucose tolerance tests, mice were fasted overnight $(16 \mathrm{~h})$ and then injected with glucose ( $2 \mathrm{~g} / \mathrm{kg}$ body weight, i.p.). Glucose was measured in the tail vein blood at $0,15,30,60$ and 120 min after injection using a glucometer (One Touch; Lifescan, Milpitas, CA, USA). For insulin tolerance tests, fed mice were injected with insulin (1 U/kg body weight i.p.; Humilin; Lilly, Indianapolis, IN, USA) and tail vein blood glucose was measured at 0,15 , 30, 60 and $120 \mathrm{~min}$ after injection.

Glucose uptake using ${ }^{18}$ F-fluorodeoxyglucose positron emission tomography Positron emission tomography (PET) scanning was performed with a dedicated small animal PET system (microPET-R4; Concorde Microsystems, Knoxville, TN, USA). Mice were starved for 18 to $20 \mathrm{~h}$ and then injected i.v. with ${ }^{18} \mathrm{~F}$-fluorodeoxyglucose (FDG) $(7.4 \mathrm{MBq}$ [200 $\mu \mathrm{Ci}]$ in $0.1 \mathrm{ml}$; Kirams, Seoul, Korea). Whole body PET imaging was started $60 \mathrm{~min}$ after ${ }^{18} \mathrm{~F}$-FDG injection and continued for $30 \mathrm{~min}$. Mice were kept anaesthetised with isoflurane $(0.5 \%$ [vol./vol.] in oxygen) and placed on a heating pad at $30^{\circ} \mathrm{C}$ for the duration of the procedure. The PET data were transformed to two-dimensional sinograms using histogramming and Fourier rebinning, and were reconstructed to tomographic data using 2-dimensional ordered subset expectation maximisation algorithm. Reconstructed PET 
images were converted into images of per cent injected dose per gram $(\% \mathrm{ID} / \mathrm{g})$, corrected for individual doses using the formula:

$\% \mathrm{ID} / \mathrm{g}=($ count per pixel $\times$ cross calibration factor $) /$ injected dose, if $1 \mathrm{ml}=1 \mathrm{~g}$

Western blot DBA/2, C57BL/6 and JYD mice were killed at 3,10 or 30 weeks of age by administering $\mathrm{CO}_{2}$. Skeletal muscles (hindlimb) and other tissues were rapidly excised, immediately frozen in liquid nitrogen and stored at $-70^{\circ} \mathrm{C}$. For in vivo phosphorylation assays of insulin receptor (IR) $\beta$ and Akt/protein kinase B (PKB), mice were fasted for $16 \mathrm{~h}$ and then injected with insulin (1 U/kg body weight, i.p.). After $30 \mathrm{~min}$, skeletal muscle tissues were quickly collected and stored in liquid nitrogen. These tissues (10-30 mg) were lysed in $1 \%$ SDS buffer (1\% SDS [wt/vol.], $50 \mathrm{mmol} / 1$ Tris$\mathrm{HCl}, \mathrm{pH} 7.4,150 \mathrm{mmol} / \mathrm{l} \mathrm{NaCl}, 1 \mathrm{mmol} / \mathrm{l}$ EDTA, $1 \mathrm{mmol} / \mathrm{l}$ $\mathrm{NaF}, 1 \mathrm{mmol} / \mathrm{l}$ phenylmethylsulfonyl fluoride) and subjected to western blotting with the following primary antibodies: anti-IR $\beta$ antibody (Upstate, Lake Placid, NY, USA), anti-IRS antibody (Upstate), anti-tubulin and anti-glucose transporter 4 (GLUT-4) antibodies (Santa Cruz Biotechnology, Santa Cruz, CA, USA), anti-Akt/PKB antibody (New England BioLabs, Ipswich, MA, USA), anti-peroxisome proliferator-activated receptor (PPAR) $\gamma$ (New England BioLabs), anti-caveolin-1 and -3 antibodies (BD Transduction, Palo Alto, CA, USA), anti-phospho-IR $\beta$ and phospho-Akt/PKB antibodies (Cell Signaling Technology, Beverly, MA, USA) and anti-flotillin antibody (BD Transduction).

Immunohistochemistry Muscle tissues were removed, fixed in Tissue-Tek (Sakura Finetek, Torrance, CA, USA) solution and cut in $4 \mu \mathrm{m}$ consecutive sections on a cryostat. The sections were immediately collected on glass slides to be used for haematoxylin-eosin and immunohistological staining. Sections were incubated overnight at $4{ }^{\circ} \mathrm{C}$ with anti-caveolin-1 antibody (1:100; BD Transduction) and anti-myogenin antibody (1:100; Santa Cruz Biotechnology). The avidin-biotin peroxidase complex method according to the LSAB kit (DAKO, Glostrup, Denmark) was used and slides were counterstained with haematoxylin. Slides were dehydrated sequentially in ethanol, cleared with xylenes and mounted with Permount.

Caveolae extraction and fractionation Caveolae were extracted according to the detergent-free protocol. Briefly, virus-infected skeletal muscle cells were washed with ice-cold phosphate-buffered saline, scraped into $1 \mathrm{ml}$ of $0.5 \mathrm{~mol} \mathrm{NaCO}_{3}$, $\mathrm{pH}$ 11.0, and homogenised. Sonicated cell samples were mixed with equal volumes of $80 \%$ (wt/vol.) sucrose solution in 2-(N-morpholino)ethanesulfonic acid (MES)-buffered saline (25 mmol/l MES, pH 6.5, $0.15 \mathrm{~mol} \mathrm{NaCl}$ ), placed in an ultracentrifuge tube and overlaid with $4 \mathrm{ml} \mathrm{30 \%}$ (wt/vol.) sucrose and $4 \mathrm{ml}$ of $5 \%$ (wt/vol.) sucrose in MES-buffered saline containing $0.25 \mathrm{~mol} \mathrm{Na}_{2} \mathrm{CO}_{3}$. Gradients were generated by centrifugation at $200,000 \mathrm{~g}$ for $18 \mathrm{~h}$ in a SW41 rotor (Beckman, Fullerton, CA, USA). Fractions that contained caveolin proteins were used as caveolar fractions and fractions that did not contain caveolin proteins were used as noncaveolar fractions.

Production of short-hairpin lenti-Cav-1 viral vector The short-hairpin RNA (shRNA) lentivirus vector for targeting the gene that encodes caveolin-1 ( $\mathrm{Cav}-1)$, named shlenti-Cav-1, was constructed by inserting synthetic double-stranded oligonucleotides (5'-CGGAATTCCATCTACAAGCCCAA CAACttcgGTTGTTGGGCTTGTAGATGTTTTTGATATC TAGACA-3') into EcoRI-XbaI restriction enzyme sites of shLenti1.1-lentiviral vector (Macrogen, Seoul, Korea). This shLenti1.1 lentiviral vector is designed to produce shRNAs from the U6 promoter and to express a gene for green fluorescent protein (GFP) located downstream from an internal ribosomal entry site (IRES). As a control vector (shlenti-GFP), scrambled sequences (AATCGCATAGCG TATGCCGTT) were inserted into the shLenti1.1 lentiviral vector described above.

Production of adenoviral caveolin-1 vector Infective recombinant adenovirus was made using the AdEasy system [19]. Recombinant adenovirus producing caveolin-1 (Ade-Cav-1) was produced by inserting the wild-type Cav-1 cDNA into a shuttle plasmid (pAdTRACK-CMV; Invitrogen, Carlsbad, CA, USA) and performing homologous recombination in $E$. coli BJ5183 with this shuttle vector and a large adenoviruscontaining plasmid (pAdEasy-1) following electroporation. Recombinant viruses were selected with kanamycin then determined by restriction endonuclease digestion. Infective adenovirus virons were produced following transfection of the linearised recombinant adenovirus plasmid in HEK293 cells. Virus stocks were amplified in HEK293 cells on $15 \mathrm{~cm}$ plates and purified using kits (BD Adeno-X Purification kits; Clontech, Palo Alto, CA, USA). A control vector (AdeGFP) carrying the cDNA for modified GFP, EGFP, was prepared as described above.

Statistics All results are presented as means \pm SEM. Statistical analyses were performed using one-way factorial ANOVA. Pair-wise comparisons were evaluated by means of Fisher's least significant differences. Statistical significance was assumed at $p<0.05$.

\section{Results}

Validation of JYD mouse model of type 2 diabetes To confirm the development of diabetes in the JYD mice, we 
compared blood glucose levels in male JYD mice and parental male C57BL/6 and DBA/2 mice. The mean blood glucose levels in male JYD mice at 10 weeks of age $(10.1 \mathrm{mmol} / \mathrm{l})$ were significantly higher than those of control male C57BL/6 (9.0 mmol/1) and DBA/2 $(8.3 \mathrm{mmol} / \mathrm{l})$ mice. At 30 weeks, blood glucose levels in male JYD mice were significantly higher than at 10 weeks of age (Fig. 1a). Blood glucose levels $>13.8 \mathrm{mmol} / 1$ [20] were considered to be diabetic. Male JYD mice showed a significantly higher $(51 \%, 24 / 47)$ incidence of diabetes at 30 weeks of age than 30 -week-old male C57BL/6 and $\mathrm{DBA} / 2$ mice $(8.7 \%, 4 / 46$ and $2.2 \%, 1 / 46$, respectively; Fig. 1b). Male JYD mice showed $10.6 \%$ incidence of diabetes even at 10 weeks of age, whereas none of the male C57BL/6 and DBA/2 mice (Fig. 1b) or female JYD mice (data not shown) developed diabetes at this age. The body weights of male JYD mice were significantly higher than those of male C57BL/6 and DBA/2 mice at 10 weeks of age, but were not significantly different at 30 weeks of age (Fig. 1c). Female JYD mice showed normal blood glucose levels and body weights at all ages tested (data not shown).

Glucose and insulin tolerance We performed glucose tolerance tests in male JYD mice at 10 and 30 weeks of age. At 10 weeks of age, blood glucose levels peaked at $15 \mathrm{~min}$ after glucose injection and were significantly higher at all time points in male JYD mice than in male C57BL/6 and DBA/2 mice (Fig. 2a). At 30 weeks of age, male JYD

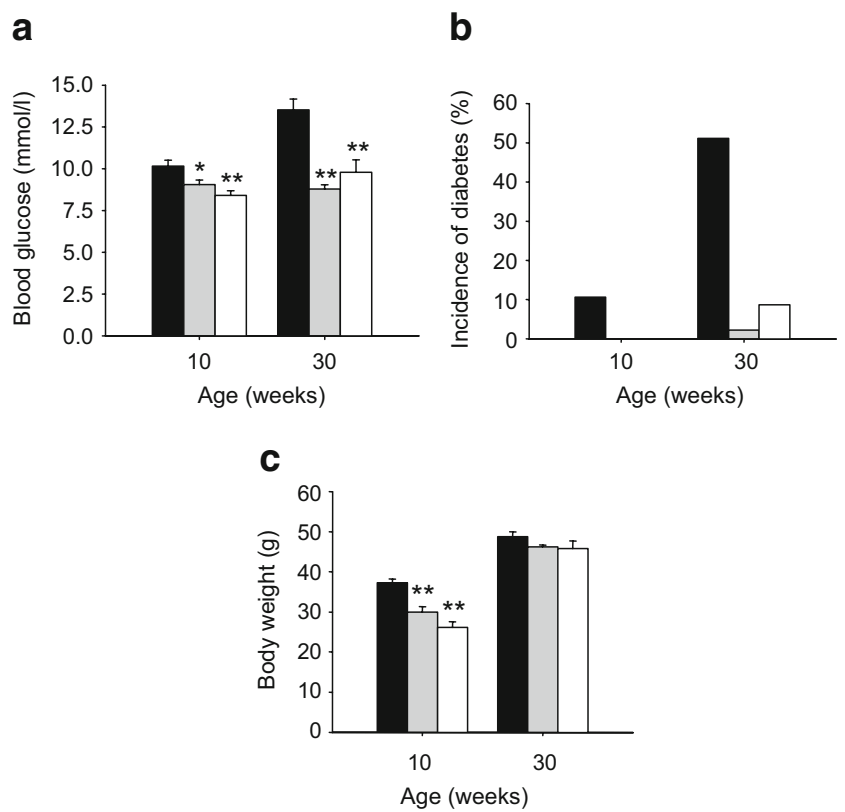

Fig. 1 Mean non-fasting blood glucose levels (a), cumulative incidence of diabetes (b) and body weights (c) of male JYD mice (black bars) at 10 and 30 weeks. Age-matched male C57BL/6 (grey bars) and DBA/2 (white bars) mice served as controls. Values are means \pm SEM, $n=45$ per group. ${ }^{*} p<0.05,{ }^{* *} p<0.01$ vs JYD mice
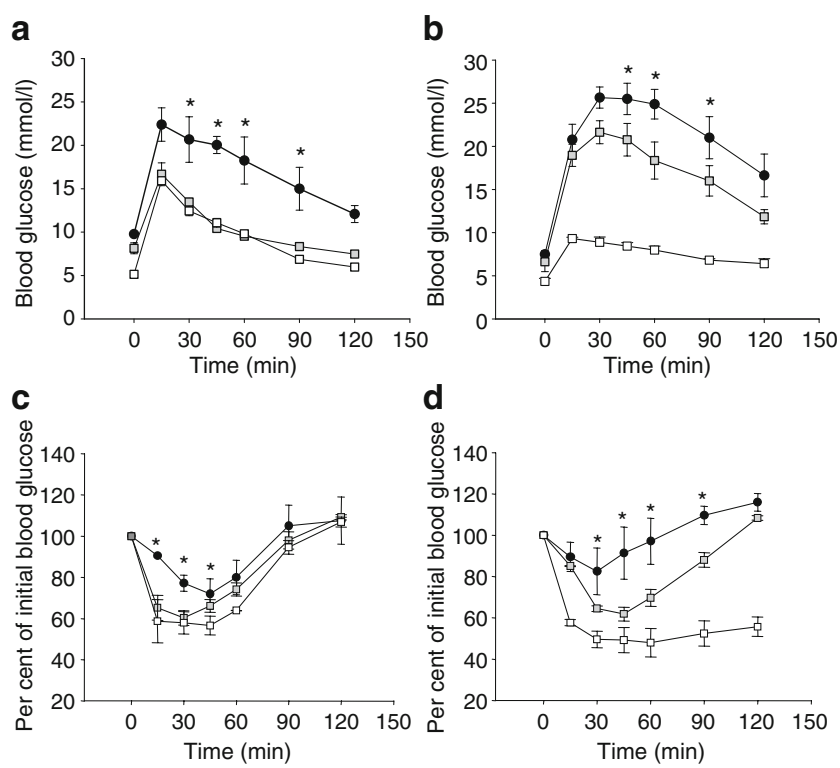

Fig. 2 Intraperitoneal glucose tolerance $(\mathbf{a}, \mathbf{b})$ and insulin tolerance $(\mathbf{c}, \mathbf{d})$ test results at $10(\mathbf{a}, \mathbf{c})$ and $30(\mathbf{b}, \mathbf{d})$ weeks of age in male JYD mice (circles). Age-matched male C57BL/6 (grey squares) and DBA/2 (white squares) mice served as controls. Values are means \pm SEM, $n=6$ per group. Representative data from three independent experiments are shown. ${ }^{*} p<0.05$ vs DBA mice

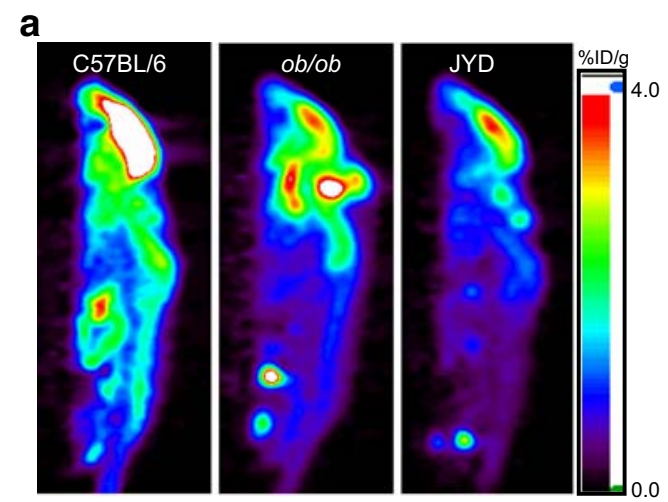

b

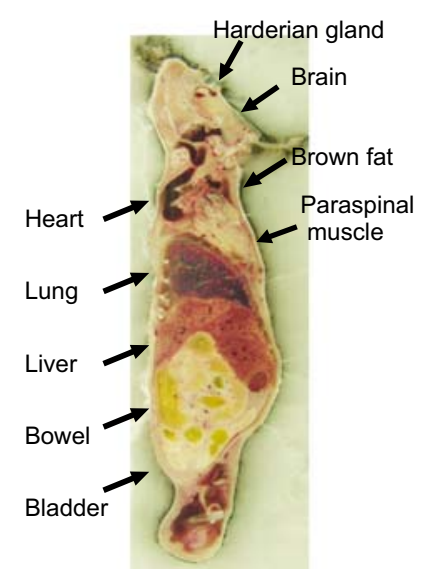

Fig. 3 Whole body ${ }^{18}$ F-FDG PET images showing glucose uptake in male JYD mice. a Sagittal sections through C57BL/6, ob/ob and JYD mice. b Corresponding cryotomy section. $\% \mathrm{ID} / \mathrm{g}$, per cent injected dose per gram 
mice showed more severe impairment of glucose tolerance than at 10 weeks of age. Male C57BL/6 mice also showed impaired glucose tolerance at 30 weeks, whereas male DBA/ 2 mice showed normal clearance of exogenous glucose (Fig. 2b). To determine whether male JYD mice have a defect in insulin responsiveness, we performed insulin tolerance tests. At 10 weeks of age, blood glucose levels in male JYD mice were not significantly reduced after insulin injection compared with male C57BL/6 and DBA/2 mice (Fig. 2c). At 30 weeks of age, male JYD mice showed little reduction of glucose in response to insulin (Fig. 2d).

FDG-PET of glucose metabolic rates It has been reported that FDG uptake in type 2 diabetes patients is poor because the body's glucose utilisation is decreased [21]. With this method, we therefore examined whole body glucose utilisation of male JYD mice, using C57BL/6 and $o b / o b$ mice as controls. FDG uptake in 30-week-old C57BL/6 mice was higher than that in JYD mice of the same age and that in 10-week-old $o b / o b$ mice (Fig. 3), especially in brain, spine and lung. The low systemic glucose uptake of JYD mice was similar to that of $o b / o b$ mice.

Levels of insulin signalling-related proteins and phosphorylated proteins in skeletal muscle To elucidate the mechanisms of age-dependent type 2 diabetes in JYD mice, we analysed the levels of insulin signalling-related proteins, such as IRS-1, IR $\beta$, Akt/PKB, PPAR- $\gamma$ and GLUT-4 in the skeletal muscle of JYD and control DBA/2, C57BL/6 mice at 3, 10 and 30 weeks of age (Fig. 4a). The levels of IRS-1, Akt/PKB and PPAR- $\gamma$ significantly decreased with age both in JYD and C57BL/6 mice, whereas levels of IR $\beta$ and GLUT-4 did not change with age either in JYD or in C57BL/6 mice (Fig. 4c,d). The levels of these proteins did not change with age in DBA/2 mice (Fig. 4b).

Impairment of insulin action might be affected by changes in activity as well as basal levels of the molecules involved in insulin signalling [22]. Therefore, we checked the phosphorylation status of IR $\beta$ and $A k t / P K B$ upon insulin stimulation in 30-week-old DBA/2, C57BL/6 and JYD mice. We found that phosphorylation of IR $\beta$ and $A k t / P K B$ after insulin stimulation was increased in DBA/2 mice, but remained unchanged in C57BL/6 mice and JYD mice (Fig. 4e).

Levels of caveolins in skeletal muscle and other insulinresponsive tissues To determine the involvement of caveolins in the mechanism for diabetes in JYD mice, we checked the levels of caveolins in skeletal muscle, adipose tissue, liver and pancreas in DBA/2, C57BL/6 and JYD mice at 3, 10 and 30 weeks of age. In all three mouse groups, quantitative western blots showed that the caveolin-1 levels were increased at 30 weeks compared with 3 weeks (Fig. 5a). However, caveolin-1 levels in skeletal muscle of DBA/2 a

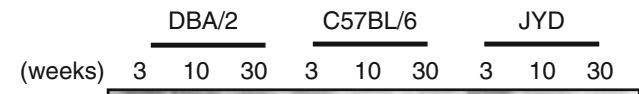

IRS-1 D m m
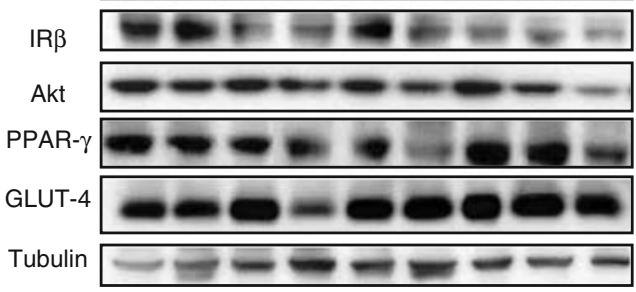

b

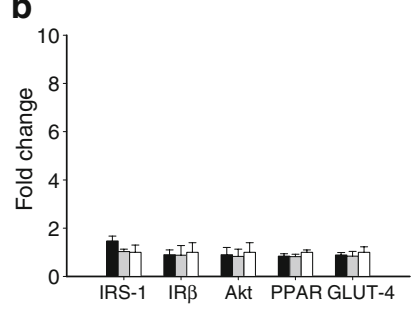

C

d
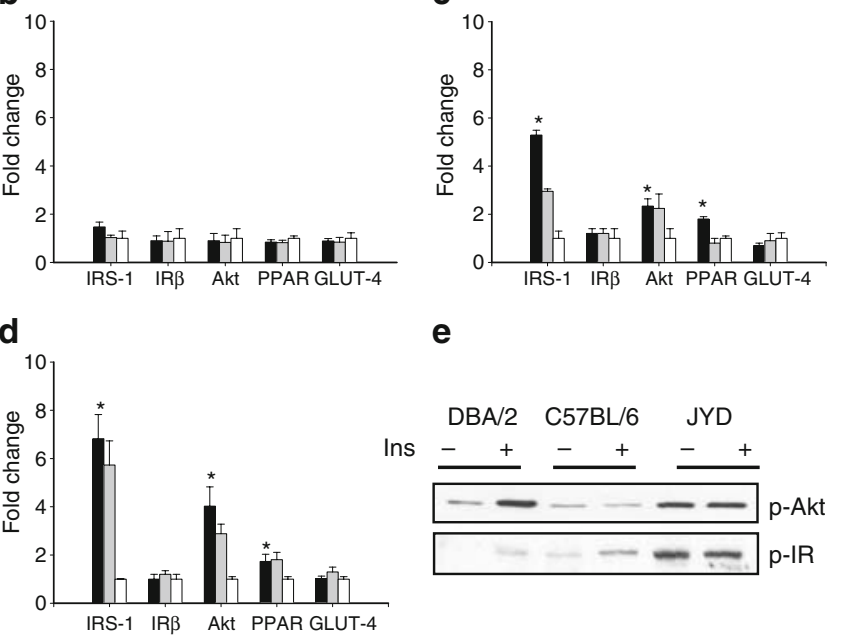

e

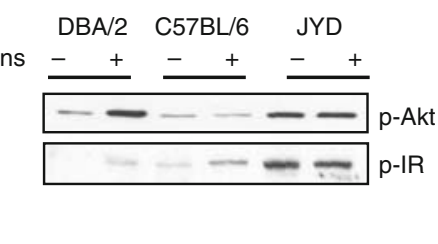

Fig. 4 Levels of insulin-related proteins and phosphorylated proteins in skeletal muscle of JYD mice. a Skeletal muscle tissue from 3-, 10and 30-week-old male DBA/2, C57BL/6 and JYD mice was isolated and levels of insulin signalling proteins analysed by western blot. Tubulin was used as loading control. b-d Quantitative analysis of western blots for DBA/2 (b), C57BL/6 (c) and JYD (d). The relative fold change in 3 weeks (black columns) to its control band (30 weeks, white columns) on western blots was quantitated by densitometric analysis. Grey bars, 10 weeks. Each bar is the mean \pm SEM. ${ }^{*} p<0.05$ vs the level at 30 weeks, $n=3$ per group. e Phosphorylated Akt (p-Akt) and phosphorylated IR $(p-I R \beta)$ in skeletal muscle tissue were determined by western blotting in 30 -week-old mice injected with $(+)$ insulin (Ins) or without (-)

and $\mathrm{C} 57 \mathrm{BL} / 6$ mice increased about eightfold, in contrast to the twofold increase seen in JYD mice (Fig. 5b). The levels of caveolin-3, the muscle-specific caveolin isoform, did not change with age in DBA/2, JYD and C57BL/6 mice.

It has been reported that ageing skeletal muscle can contain adipose tissue [23]. Therefore, to rule out that caveolin-1 production detected in skeletal muscle originated from adipocytes within ageing tissues, we examined the expression of Lep (an adipogenic gene that encodes leptin) and Myog (which encodes myogenin, a skeletal muscle marker) by RT-PCR in skeletal muscle tissues of 30-week-old mice (see Electronic supplementary material [ESM 1]). As shown in ESM Fig. 1a, expression of Lep was detected only in adipose tissues, but not in skeletal muscle, whereas Myog 
was expressed only in skeletal muscle tissues. These results suggest that the caveolin-1 detected in muscle did not originate from adipose tissue. In addition, immunohistochemical analysis showed that caveolin-1 staining of skeletal muscle fibre was lower in 30-week-old JYD mice than in C57BL/6 and DBA/2 mice (ESM Fig. 1b), which was similar to the pattern of caveolin-1 protein levels seen by western blot (Fig. 5a,b).

Also, we checked the caveolin-1 level in non-diabetic F1 male and female mice. As seen in Fig. 5c, we found that the level of caveolin-1 in these non-diabetic mice was higher than in the mice showing diabetic phenotype.

Downregulation of caveolin-1 production by lentivirusmediated caveolin-1 silencing impairs glucose tolerance and insulin sensitivity in C57BL/6 mice Since C57BL/6 and DBA/2 mice had significantly high levels of caveolin-1

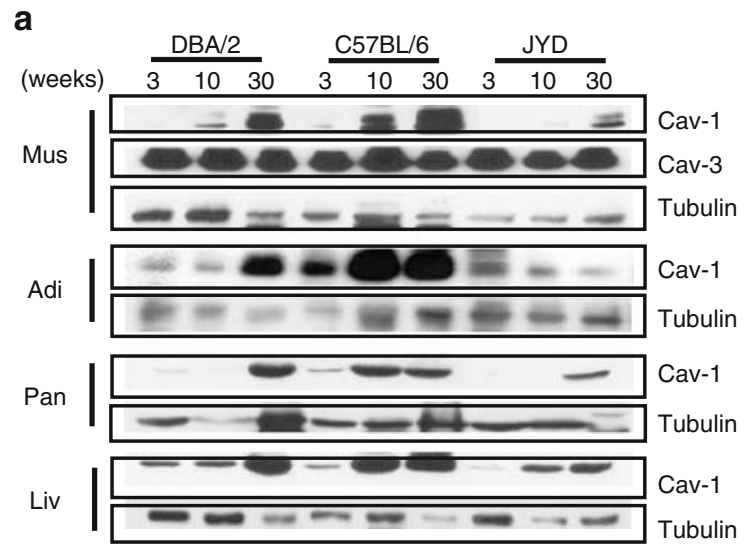

b

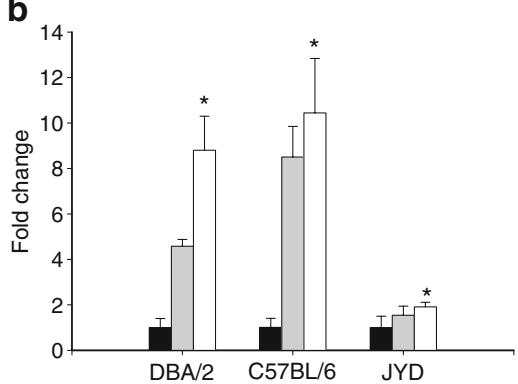

C

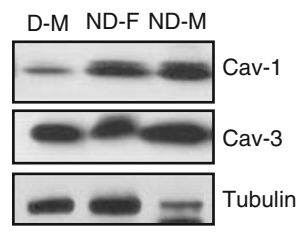

Fig. 5 Levels of caveolins in skeletal muscle and other insulinresponsive tissues. Tissues from mice were isolated and analysed for levels of caveolin proteins by western blotting. a Levels of caveolin-1 (Cav-1) and caveolin-3 (Cav-3) at 3, 10 and 30 weeks in skeletal muscle (Mus), adipose tissue (Adi), Pancreas (Pan) and Liver (Liv) of DBA/2, C57BL/6 and JYD mice. Tubulin was used to confirm equal loading. b Quantitative analysis of western blots of caveolin-1 in skeletal muscle from mice as indicated. The relative fold change in 30 weeks (white bars) to its control band (3 weeks, black bars) on western blots was quantitated by densitometric analysis. Grey bars, 10 weeks. Each bar is the mean \pm SEM. ${ }^{*} p<0.05$ vs the level at 3 weeks, $n=3$ per group. c Levels of caveolin in skeletal muscle tissues from diabetic and non-diabetic F1 JYD mice. D-M, diabetic male; ND-F, non-diabetic female; ND-M, non-diabetic male compared with JYD mice (Fig. 5), we asked whether downregulation of caveolin-1 production would negatively affect glucose and insulin tolerance in C57BL/6 mice. To answer this question, we created a lentivirus expressing an shRNA that inhibits caveolin-1 production (shlenti-Cav-1) and GFP (shlenti-GFP) as a marker (ESM Fig. 2). We first assessed the efficiency of lentivirus-mediated caveolin-1 silencing by infecting L6 cells in vitro (Fig. 6a). Western blotting showed that caveolin-1 production was reduced as a result of shlenti-Cav-1 infection (Fig. 6b). When we examined the caveolar/non-caveolar compartments after sucrose gradient fractionation, we found that the caveolin1 level in the caveolar compartment was reduced in cells infected with shlenti-Cav-1 (Fig. 6c). As seen in Fig. 6d, immunohistochemistry revealed a decrease in the caveolin1 protein level compared with shlenti-GFP-treated mice. Since the efficiency of shlentivirus production decreased 2 weeks after injection (data not shown), we conducted the experiment up until 2 weeks after viral injection. At 14 days after the injections, the caveolin-1-downregulated mice showed more severe impairment of glucose (Fig. 6e) and insulin tolerance (Fig. 6f) than shlenti-GFP-treated mice.

Adenoviral-mediated caveolin-1 overproduction improves glucose and insulin tolerance and glucose uptake in 30-week-old JYD mice Since JYD showed significantly lower caveolin-1 levels than C57BL/6 mice (Fig. 5), we asked whether overproduction of caveolin-1 would positively affect glucose and insulin tolerance in 30-week-old JYD mice. We used an adenoviral vector producing both caveolin-1 and enhanced GFP (Ade-Cav-1) and an adenoviral vector producing enhanced GFP as a control (Ade-GFP; ESM Fig. 3a). We first assessed the efficiency of adenoviral-mediated overproduction of caveolin-1 by infecting L6 cells in vitro (Fig. 7a). Western blotting showed that caveolin-1 production increased in Ade-Cav-1-infected cells compared with Ade-GFPinfected cells (Fig. 7b). By sucrose gradient fractionation, we found that the caveolin-1 content of the caveolar compartment was increased in cells infected with Ade-Cav1 (Fig. 7c). To determine whether intra-muscular injection of the adenoviral vector would affect other tissues, we injected Ade-GFP $\left(3 \times 10^{11}\right.$ plaque-forming units $)$ intra-muscularly and examined the muscle, adipose tissue and liver. Western blot (ESM Fig. 3b) showed that GFP protein was confined to the muscle after intra-muscular injections, but was found in all tissues after intra-venous injection. When Ade-Cav-1 was injected into the skeletal muscle of JYD mice, immunohistochemical staining of the muscle revealed that caveolin-1 production was increased compared with Ade-GFP-injected mice (Fig. 7d). Using an in vivo fluorescence imaging system, we observed that the production of caveolin-1 was maximal at 3 days after injection (data not shown); therefore, subsequent experiments were performed at day 3 . 
a

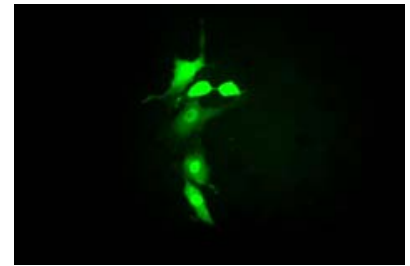

Shlenti-GFP

b

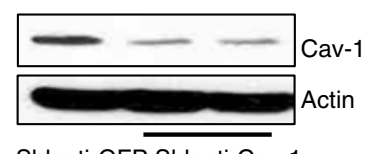

Shlenti-GFP Shlenti-Cav-1

d

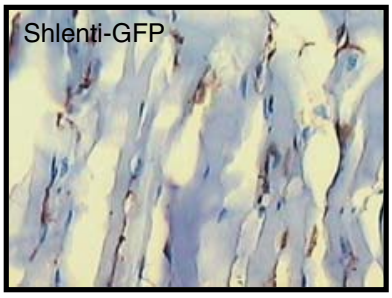

e
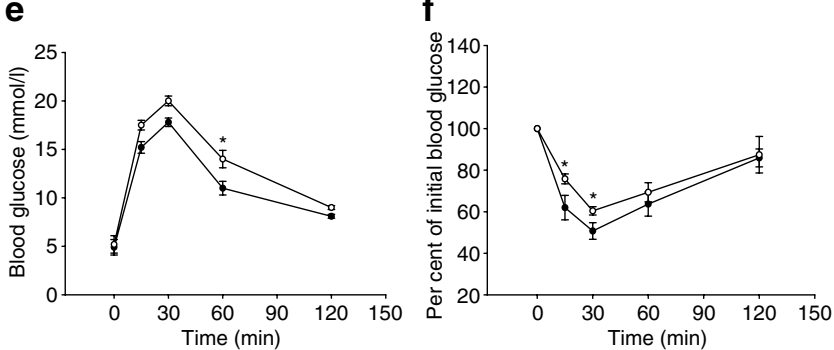

Fig. 6 Impairment of glucose and insulin tolerance in shlenti-Cav-1injected C56BL/6 mice. a L6 cells were infected with the indicated lentivirus and observed under the fluorescence microscope. b Western blot analysis of caveolin-1 protein production (Cav-1) in infected L6 cells after transduction. Actin level was used as a control. c ShlentiGFP and shlenti-Cav-1 virus-infected skeletal muscle cells were homogenised without detergent, sonicated and subjected to sucrose density gradient fractionation. The level of caveolin-1 was analysed by western blotting; flotillin level was used as a lipid raft marker. Cav, caveolae; N-C, non-caveolae. d The hindlimb of 30-week-old C57BL/ 6 mice was injected with shlenti-Cav-1 or shlenti-GFP at a multiplicity of infection (MOI) of $3 \times 10^{7}$, and caveolin-1 production was analysed by immunohistochemistry. e,f Intraperitoneal glucose tolerance (e) and insulin tolerance test (f) results at 2 weeks after injection of shlentiGFP (black circles) and shlenti-Cav-1 virus (white circles). Values are means \pm SEM, $n=5$ per group. ${ }^{*} p<0.05$ vs shlenti-GFP

After glucose loading, exogenous glucose was more efficiently cleared in the Ade-Cav-1-injected animals than in Ade-GFP-injected animals (Fig. 7e). Also, exogenously injected insulin significantly reduced glucose level in AdeCav-1-treated mice compared with Ade-GFP-treated mice, suggesting that insulin sensitivity was improved (Fig. 7f).
Ade-GFP and Ade-Cav-1 were injected into each side of the thigh muscle of JYD mice (Fig. 7g) and glucose uptake checked using FDG-PET. Glucose uptake increased in AdeCav-1-injected muscle compared with Ade-GFP-injected muscle at 3 days after injection (Fig. $7 \mathrm{~g}, \mathrm{~h}$ ).

\section{Discussion}

We show here that male JYD mice develop moderate to severe hyperglycaemia in old age and are not obese as compared with the parental C57BL/6 and DBA/2 strains. The JYD mouse seems to be a promising model to study the role of ageing in the development of the late-onset diabetic type 2 diabetes phenotype.

Skeletal muscle is the primary sink for insulin-mediated glucose uptake, whereas adipose tissue is relatively inert and accounts for only 2 to $3 \%$ of total glucose uptake [24]. Many studies examining the role of ageing on glucose uptake have demonstrated decreased muscular insulin sensitivity [25, 26]. These studies suggest that insulin resistance in elderly people appears predominantly in the skeletal muscle, but the precise mechanism underlying age-dependent glucose uptake is not known. We therefore attempted to address this issue, employing the JYD mouse model.

When peripheral tissues are resistant to insulin, glucose in the blood cannot be effectively absorbed and metabolised in tissues, resulting in hyperglycaemia. In male JYD mice, exogenous glucose was not cleared effectively and exogenous insulin did not effectively lower blood glucose levels, compared with DBA/2 mice. Interestingly, C57BL/6 mice also showed impaired glucose tolerance and slightly impaired insulin response at 30 weeks of age. However, C57BL/6 mice did not develop hyperglycaemia, in contrast to male JYD mice.

Insulin stimulates IRs, which activate kinases such as IR tyrosine kinase, phosphatidylinositol 3-kinase and Akt/PKB $[27,28]$. Activation of this signal transduction pathway increases glucose transport through GLUT-4 and glucose metabolism. Thus, decreased levels of any of the molecules in this pathway or their malfunction could result in insulin resistance [29]. It is well known that a single defect in the insulin-signalling pathway, such as knockout of the IR, IRS-2 [30] or Akt [31], can produce type 2 diabetes.

To elucidate the mechanisms of ageing-dependent type 2 diabetes, we first analysed insulin signalling molecules in skeletal muscle tissues. We found that 30-week-old C57BL/6 and JYD mice show impaired glucose and insulin tolerance and that this phenotype could partially be explained by the low levels of IRS-1, Akt/PKB and PPAR- $\gamma$, and by the low levels of phosphorylated IR $\beta$ and $\mathrm{Akt} / \mathrm{PKB}$ upon insulin stimulation found in the muscle tissue of these mice. However, the incidence of 
a

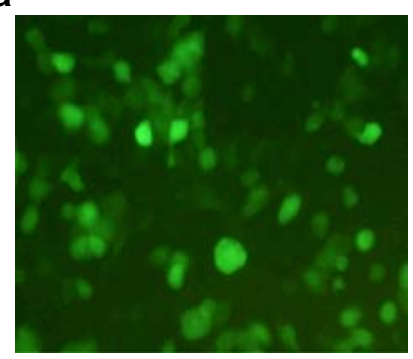

Ade-GFP

b

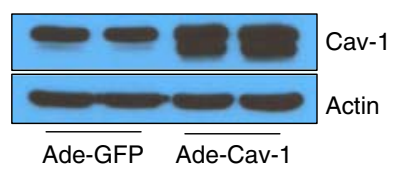

d

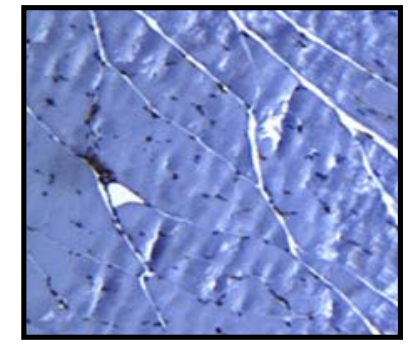

Ade-GFP

e

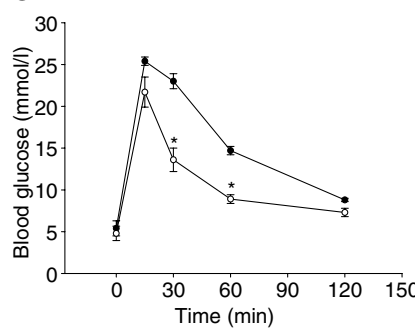

g

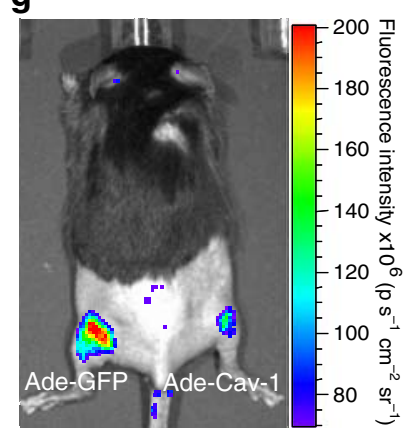

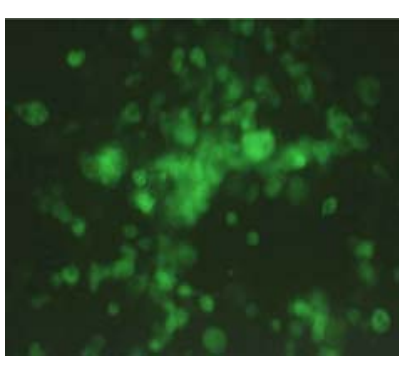

Ade-Cav-1

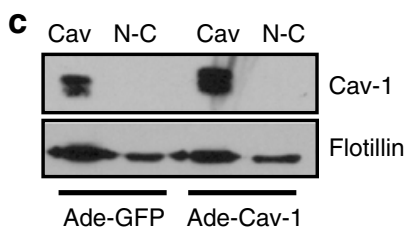

Ade-Cav-1

f

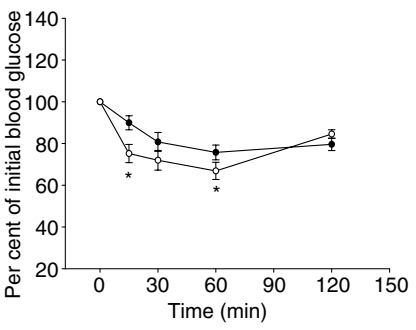

h

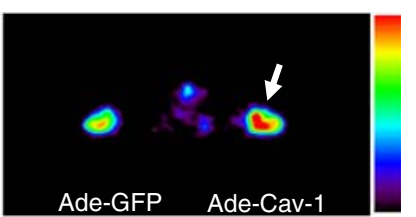

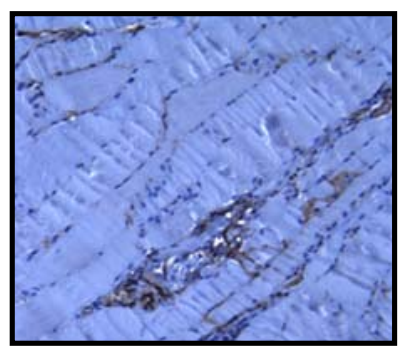

4Fig. 7 Improvement of glucose and insulin tolerance in Ade-Cav-1injected JYD mice. a L6 cells were infected and observed under the fluorescence microscope. $\mathbf{b}$ Western blot analysis of caveolin-1 protein production (Cav-1) in L6 cells after transduction. Actin was analysed as a control. c Ade-GFP and Ade-Cav-1 virus-infected skeletal muscle cells were homogenised without detergent, sonicated and subjected to sucrose density gradient fractionation. The level of caveolin-1 was analysed by western blotting, with flotillin level used as a lipid raft marker. Cav, caveolae; N-C, non-caveolae. d The hindlimbs of 30week-old diabetic JYD mice were injected with Ade-Cav-1 or AdeGFP $\left(3 \times 0^{11}\right.$ plaque-forming units) and caveolin-1 production at 30 weeks detected by immunohistochemistry. e, f Three days after adenovirus injection, mice were fasted for $18 \mathrm{~h}$, and glucose tolerance (e) and insulin sensitivity (f) tests were performed. Black circles, AdeGFP; open circles, Ade-Cav-1. Data are means \pm SEM. ${ }^{*} p<0.05$ vs Ade-GFP group, $n=6$ per group. $\mathrm{g}$ Transduction efficiency of skeletal muscle regions of control (Ade-GFP) or caveolin-1 (Ade-Cav-1)injected mice. Fluorescence intensity is shown as $\mathrm{p} \mathrm{s}^{-1} \mathrm{~cm}^{-2} \mathrm{sr}^{-1}$, where $p$ stands for photons and $s r$ represents surface. $\mathbf{h}$ Glucose uptake was measured with ${ }^{18}$ F-FDG-PET. Red, high uptake; black, low uptake. Images are transverse sections through Ade-GFP- or AdeCav-1-injected JYD mice. Arrow, high uptake

the senescent phenotype [32]. Therefore, we examined whether expression of caveolin shows age-dependent changes in JYD mice compared with their parental strains. Caveolin-3 levels did not change with age in any of these strains of mice. Interestingly, all strains showed an agerelated increase in skeletal muscle caveolin-1, but the increase in C57BL/6 and DBA/2 mice was substantially higher than that in JYD mice. These results suggest that a potential defect in caveolin-1 content may be a factor in the development of type 2 diabetes and insulin sensitivity in the JYD mouse model. Consistent with this, caveolin-1-deficient mice show insulin resistance and defective IR protein content in adipose tissue [10].

Although other tissues showed changes in caveolin-1 levels with age that were similar to those for skeletal muscle in our study, we focused our subsequent experiments on manipulating caveolin-1 levels in skeletal muscle because of the importance of muscle insulin resistance in type 2 diabetes and our previous results on the role of caveolin-1 in glucose uptake in skeletal muscle [18].

To test the role of caveolin-1 in the development of insulin sensitivity, we experimentally reduced caveolin-1 levels in C57BL/6 mice and increased the caveolin-1 levels in JYD mice. When caveolin-1 production was downregulated in $\mathrm{C} 57 \mathrm{BL} / 6$ mice by RNA interference, blood glucose levels increased and glucose tolerance and insulin sensitivity were impaired compared with control C57BL/6 mice. In contrast, when caveolin-1 was overproduced in JYD mice, glucose tolerance and insulin sensitivity improved and glucose uptake increased in skeletal muscles. We also checked the caveolin-1 levels of C57BL/6× DBA $/ 2$ F1 hybrid mice with normal blood glucose levels and found that the level of caveolin-1 in these mice was higher than in F1 mice showing the diabetic phenotype. These results 
strongly suggest a correlation between the level of caveolin-1 in the skeletal muscle and the development of the diabetic phenotype of JYD mice.

Glucose transport and glucose utilisation in cells are involved in the pathogenesis of insulin resistance [18, 29, 33]. We previously found that GLUT-4 was mainly localised in caveolin-rich membrane domain [18, 34] and that caveolin-1 is involved in insulin-dependent glucose uptake via regulation of GLUT-4 translocation in muscle cells rather than via changes jin the level of GLUT-4. It is known that caveolin-1 participates in cellular trafficking and caveolae contain many molecules, including soluble $\mathrm{N}$-ethylmaleimidesensitive factor attachment receptor proteins $[35,36]$, that are required for the final fusion of GLUT- 4 vesicles by integrating GLUT-4 proteins into the plasma membrane. Therefore, it is possible that caveolin-1 could affect GLUT4 translocation by regulation of these molecules. In addition, upregulation of caveolin-1 in muscle by exercise is an important molecular response that influences insulin sensitivity [37]. These observations support the concept that elevated levels of caveolin-1 enhance overall insulin sensitivity via the glucose uptake system.

We compared the diabetic phenotype of JYD mice with age-independent type 2 diabetic $o b / o b$ mice. For this, we checked the patterns of production of insulin signalling molecules as well as caveolins in $o b / o b$ mice. Interestingly, obese diabetic $o b / o b$ mice, in which GLUT-4 protein production was downregulated (data not shown), showed negligible changes in the level of caveolin-1 compared with control mice.

In conclusion, we have shown that the JYD mouse is an appropriate model for studying ageing-dependent nonobese type 2 diabetes. We have also shown that downregulation of caveolin-1 production in the skeletal muscle correlates with the development of type 2 diabetes in old JYD mice, and that insulin sensitivity in these mice is regulated by caveolin-1 production. It can therefore be speculated that induction of caveolin production in the skeletal muscle and control of insulin sensitivity in the aged could prevent or delay the appearance of type 2 diabetes.

Acknowledgements This study was supported by the grants from the Aging and Apoptosis Research Center of the Korea Science and Engineering Foundation (RII-2002-097-05001-0, RII-2002-09700001-0), the Radiation Medicine Research Center (from the Ministry of Science \& Technology through the National Nuclear Technology Program) and the Innovative Research Institute for Cell Therapy (A062260), Korea.

We thank A. Kyle for editorial assistance. We also thank K. Sun Woo for the excellent technical assistance, and K. Min Kim and J. Hyun Kang for their laborious imaging analysis.

Duality of interest The authors declare that there is no duality of interest associated with this manuscript.

\section{References}

1. Saltiel AR (2001) New perspectives into the molecular pathogenesis and treatment of type 2 diabetes. Cell 104:517-529

2. Scheen AJ (2005) Diabetes mellitus in the elderly: insulin resistance and/or impaired insulin secretion? Diabetes Metab 31 (Spec No 2):5S27-5S34

3. Kim DJ, Song KE, Park JW, Cho HK, Lee KW, Huh KB (2007) Clinical characteristics of Korean type 2 diabetic patients in 2005 . Diabetes Res Clin Pract 77(Suppl 1):S252-S257

4. Park SY, Choi GH, Choi HI, Ryu J, Jung CY, Lee W (2005) Calorie restriction improves whole-body glucose disposal and insulin resistance in association with the increased adipocyte-specific GLUT4 expression in Otsuka Long-Evans Tokushima fatty rats. Arch Biochem Biophys 436:276-284

5. Kanemoto N, Hishigaki H, Miyakita A et al (1998) Genetic dissection of "OLETF", a rat model for non-insulin-dependent diabetes mellitus. Mamm Genome 9:419-425

6. Sargiacomo M, Scherer PE, Tang Z et al (1995) Oligomeric structure of caveolin: implications for caveolae membrane organization. Proc Natl Acad Sci U S A 92:9407-9411

7. Schlegel A, Lisanti MP (2000) A molecular dissection of caveolin-1 membrane attachment and oligomerization. Two separate regions of the caveolin-1 C-terminal domain mediate membrane binding and oligomer/oligomer interactions in vivo. $\mathrm{J}$ Biol Chem 275:21605-21617

8. Song KS, Scherer PE, Tang Z et al (1996) Expression of caveolin-3 in skeletal, cardiac, and smooth muscle cells. Caveolin-3 is a component of the sarcolemma and co-fractionates with dystrophin and dystrophin-associated glycoproteins. J Biol Chem 271:15160-15165

9. Cohen AW, Combs TP, Scherer PE, Lisanti MP (2003) Role of caveolin and caveolae in insulin signaling and diabetes. Am J Physiol Endocrinol Metab 285:E1151-E1160

10. Cohen AW, Razani B, Wang XB et al (2003) Caveolin-1-deficient mice show insulin resistance and defective insulin receptor protein expression in adipose tissue. Am J Physiol Cell Physiol 285: C222-C235

11. Cho KA, Ryu SJ, Park JS et al (2003) Senescent phenotype can be reversed by reduction of caveolin status. J Biol Chem 278:2778927795

12. Cho KA, Ryu SJ, Oh YS et al (2004) Morphological adjustment of senescent cells by modulating caveolin-1 status. J Biol Chem 279:42270-42278

13. Park WY, Park JS, Cho KA et al (2000) Up-regulation of caveolin attenuates epidermal growth factor signaling in senescent cells. J Biol Chem 275:20847-20852

14. Kawabe JI, Grant BS, Yamamoto M, Schwencke C, Okumura S, Ishikawa Y (2001) Changes in caveolin subtype protein expression in aging rat organs. Mol Cell Endocrinol 176:91-95

15. Li WP, Liu P, Pilcher BK, Anderson RG (2001) Cell-specific targeting of caveolin-1 to caveolae, secretory vesicles, cytoplasm or mitochondria. J Cell Sci 114:1397-1408

16. Galbiati F, Volonte D, Engelman JA, Scherer PE, Lisanti MP (1999) Targeted down-regulation of caveolin-3 is sufficient to inhibit myotube formation in differentiating $\mathrm{C} 2 \mathrm{C} 12$ myoblasts. Transient activation of $\mathrm{p} 38$ mitogen-activated protein kinase is required for induction of caveolin-3 expression and subsequent myotube formation. J Biol Chem 274:30315-30321

17. Capozza F, Cohen AW, Cheung MW et al (2005) Muscle-specific interaction of caveolin isoforms: differential complex formation between caveolins in fibroblastic vs muscle cells. Am J Physiol Cell Physiol 288:C677-C691

18. Oh YS, Cho KA, Ryu SJ et al (2006) Regulation of insulin response in skeletal muscle cell by caveolin status. J Cell Biochem 99:747-758 
19. He TC, Zhou S, da Costa LT, Yu J, Kinzler KW, Vogelstein B (1998) A simplified system for generating recombinant adenoviruses. Proc Natl Acad Sci U S A 95:2509-2514

20. Khil LY, Jun HS, Kwon H et al (2007) Human chorionic gonadotropin is an immune modulator and can prevent autoimmune diabetes in NOD mice. Diabetologia 50:2147-2155

21. Voipio-Pulkki LM, Nuutila P, Knuuti MJ et al (1993) Heart and skeletal muscle glucose disposal in type 2 diabetic patients as determined by positron emission tomography. J Nucl Med 34:2064-2067

22. Bruning JC, Michael MD, Winnay JN et al (1998) A muscle-specific insulin receptor knockout exhibits features of the metabolic syndrome of NIDDM without altering glucose tolerance. Mol Cell 2:559-569

23. Taylor-Jones JM, McGehee RE, Rando TA, Lecka-Czernik B, Lipschitz DA, Peterson CA (2002) Activation of an adipogenic program in adult myoblasts with age. Mech Ageing Dev 123:649-661

24. Baron AD, Laakso M, Brechtel G, Edelman SV (1991) Reduced capacity and affinity of skeletal muscle for insulin-mediated glucose uptake in noninsulin-dependent diabetic subjects. Effects of insulin therapy. J Clin Invest 87:1186-1194

25. Broughton DL, Taylor R (1991) Review: deterioration of glucose tolerance with age: the role of insulin resistance. Age Ageing 20:221-225

26. Ryan AS (2000) Insulin resistance with aging: effects of diet and exercise. Sports Med 30:327-346

27. Kanzaki M, Pessin JE (2003) Insulin signaling: GLUT4 vesicles exit via the exocyst. Curr Biol 13:R574-576
28. Saltiel AR, Kahn CR (2001) Insulin signalling and the regulation of glucose and lipid metabolism. Nature 414:799-806

29. Nandi A, Kitamura Y, Kahn CR, Accili D (2004) Mouse models of insulin resistance. Physiol Rev 84:623-647

30. Withers DJ, Gutierrez JS, Towery H et al (1998) Disruption of IRS-2 causes type 2 diabetes in mice. Nature 391:900-904

31. Cho H, Mu J, Kim JK et al (2001) Insulin resistance and a diabetes mellitus-like syndrome in mice lacking the protein kinase Akt2 (PKB beta). Science 292:1728-1731

32. Cho KA, Park SC (2005) Caveolin-1 as a prime modulator of aging: a new modality for phenotypic restoration? Mech Ageing Dev 126:105-110

33. Shulman GI (2000) Cellular mechanisms of insulin resistance. J Clin Invest 106:171-176

34. Barros RP, Machado UF, Warner M, Gustafsson JA (2006) Muscle GLUT4 regulation by estrogen receptors ERbeta and ERalpha. Proc Natl Acad Sci U S A 103:1605-1608

35. Schnitzer JE, Liu J, Oh P (1995) Endothelial caveolae have the molecular transport machinery for vesicle budding, docking, and fusion including VAMP, NSF, SNAP, annexins, and GTPases. J Biol Chem 270:14399-14404

36. Liu P, Rudick M, Anderson RG (2002) Multiple functions of caveolin-1. J Biol Chem 277:41295-41298

37. Oh YS, Kim HJ, Ryu SJ et al (2007) Exercise type and muscle fiber specific induction of caveolin-1 expression for insulin sensitivity of skeletal muscle. Exp Mol Med 39:395-401 